\title{
Path Planning for Particle Obstacle Avoidance in Potential Flow Field
}

\author{
Zihao Chen ${ }^{1,2}$, Liangfu Peng ${ }^{1,2^{*}}$, Yifan Wang ${ }^{1}$ \\ ${ }^{1}$ College of Electrical \& Information Engineering, Southwest Minzu University, Chengdu 610041, China \\ ${ }^{2}$ Guangxi Key Laboratory of Precision Navigation Technology and Application, Guilin University of Electronic \\ Technology, Guilin 541004, China \\ Email: $1275153536 @ q q . c o m$
}

\begin{abstract}
The artificial potential field uses the combined force of gravitational and repulsive forces to plan the motion path of the object. There is an area trapped in a local minimum and trap vibration. At the same time, it is difficult to predict and avoid the impact caused by moving obstacles. The complex potential field of the planar flow field is established in the planning space. The potential flow theory is used to combine multiple dynamic, static obstacles and the flow field lines formed by the target point. The modified reset potential and the complex velocity are superimposed, which can effectively avoid movement over a long distance obstacle. Add eddy currents and establish temporary target points to guide particles to escape from local minimum points and multi-obstacle trap areas. Simulation results show that the particle moves more flexibly in the flow field environment, which can avoid the occurrence of motion interruption and form a safer and smoother path.
\end{abstract}

Keywords: complex potential function, complex velocity, temporary target point, obstacle environment analysis

\section{Introduction}

Path planning is an important subject in the field of intelligent research. The planning algorithm depends on the different environment in which the object is located. How to avoid various morphological obstacles in the space and smoothly reach the specified target point without motion interruption is the key research direction.

The current path planning algorithms are mainly the combination of ant colony algorithm and genetic algorithm, improved artificial potential field method and artificial flow field method ${ }^{[1]}$. Among them, the potential field and flow field algorithms have better real-time performance, fewer iterations, and can make path adjustments at the current moment in complex environments. In the flow field environment, the perception of dynamic obstacles will be more sensitive, and there is no need for obstacles to provide repulsive factors. However, there are still limitations in the flow field environment, which can be summarized as follows:

First, trap areas and local minimums trapped in the flow field; second, the potential velocity at the source point is close to infinity; third, it is not possible to effectively avoid moving obstacles in advance at long distances. In different field environments, the modification of the repulsive force field coefficient and the side-by-side algorithm can be used to solve the local minimum problem, but the surrounding environment cannot be detected in real time. At the same time, there is also the problem that the action distance is too long. Modifying the complex potential function and adding the influence of the vortex can plan a more perfect path, but it cannot effectively avoid the trap area surrounded by multiple obstacles. In view of the above common problems, this article will modify the complex potential function in fluid mechanics during fluid flow, and meet the requirements of path planning. Combined with the concept of virtual target points in the artificial potential field, for the U-shaped and C-shaped traps composed of multiple obstacles, a recognition mechanism is added to the algorithm to gradually guide the particles out of the trap area. The characteristics of the two potential fields are used to plan and modify the particle trajectories $^{[2]}$. 


\section{Environmental Model of Particles in Flow Field ${ }^{[3]}$}

Suppose it is located in the flow field environment, the source point is the target point of the mass point, and the intensity of the source point is the attractive force. There are fixed obstacles and moving obstacles in the periphery, and the flow field lines formed by the positions and motion states of the two in the flow field serve as repulsive forces and act on the moving mass points. The distribution of the unmodified environmental model and flow field lines is shown in Figure 1:

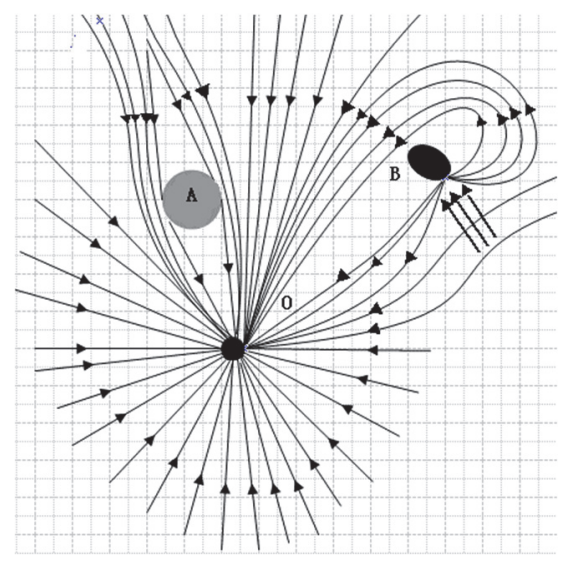

Figure 1. Flow field conceptual model.

The mass point is located at any position in the flow field environment. It is affected by gravity and converges to the source point $\mathrm{O}$, bypassing the static obstacle $\mathrm{A}$ and the dynamic obstacle $\mathrm{B}$ in the figure. Dynamic obstacle B moves along the axis.

\section{Improvement and Transformation of Complex Potential Functions}

\subsection{Complex Potential Consisting of Multiple Target Points and Static Obstacles ${ }^{[4]}$}

The flow field is composed of a large number of sources, and dipoles are formed at the same time. When the fixed obstacle is located at $z_{j}=x_{j}+i y_{j}$, the source target field strength is $E$, located at $z_{k}=x_{k}+i y_{k}$, and the virtual target point field strength $E_{i}$ is assumed to be located at $z_{i}=x_{i}+i y_{i}$. According to the potential function of the source function, the superposed complex potential function is obtained as:

$$
W(z)=f(z)+\bar{f}_{1}\left(\frac{R^{2}}{z}\right)+\bar{f}_{2}\left(\frac{R^{2}}{z}\right)
$$

where $R$ is the radius of the circular obstacle, target source complex potential is $f(z)$

$$
f(z)=\frac{E}{2 \pi} \ln \left(z-z_{i}\right), i \in(1, n), 1 \leq k \leq n
$$

Among them, the complex potential conjugate functions of the target source and $N$ virtual target sources are:

$$
\begin{gathered}
\bar{f}_{1}\left(\frac{R^{2}}{z}\right)=\frac{E}{2 \pi}\left[\ln \left(-z_{k}\right)+\ln \left(z+\frac{R^{2}}{z_{k}}\right)-\ln \left(z-z_{j}\right)\right] \\
\bar{f}_{2}\left(\frac{R^{2}}{z}\right)=\sum_{i=1}^{n} \frac{E_{i}}{2 \pi}\left[\ln \left(-z_{i}\right)+\ln \left(z+\frac{R^{2}}{z_{i}}\right)-\ln \left(z-z_{i}\right)\right]
\end{gathered}
$$

The constant terms $\ln \left(-z_{k}\right)$ and $\ln \left(-z_{i}\right)$ can also be omitted in complex potentials. 


\subsection{Cylindrical Flow and Eddy Current Superposition}

There is a local minimum for the motion of the particle under the flow field. On the basis of the acyclic cylindrical flow formed by the non-uniform flow, a point vortex is added to effectively avoid the point where the velocity vector is zero after determining the position of the stagnation point. Set the center of the fixed obstacle to the center of the point vortex ${ }^{[6]}$.

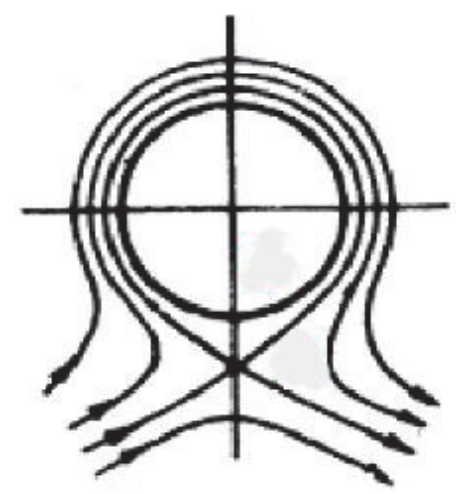

Figure 2. Circulating Cylindrical Flow

The eddy current intensity is set to $\Gamma$, and the effect after superimposed on the target source is shown in Figure 2. The reset potential after the superposition of an annulus cylinder and the clockwise point vortex is:

$$
H(z)=\frac{E}{2 \pi} \ln \left(z-z_{j}\right)+i \frac{\Gamma}{2 \pi} \ln \left(z-z_{j}\right)+c
$$

Choose a suitable complex constant $c$ such that the circumference is a streamline. When $c=-\frac{i \Gamma}{2 \pi} \ln R$, the circular flow around the cylindrical obstacle of radius $R$ has the reset potential:

$$
H(z)=\frac{E}{2 \pi} \ln \left(z-z_{j}\right)+i \frac{\Gamma}{2 \pi} \ln \left(\frac{z-z_{j}}{R}\right)
$$

At this time, the eddy current loop of the model is counterclockwise, including a stagnation point, which satisfies $\frac{\Gamma}{4 \pi U a}>1$, the stagnation point does not remain on the obstacle but enters the flow field. Because the vortex in the $90^{\circ}$ direction and the velocity direction of the flow field are the same, the resultant velocity is not zero, which can effectively avoid falling into a minimum value.

\subsection{Complex Potential Composed of Dynamic Obstacles}

Dipoles form moving obstacles, with circles as the boundary, and their reset potential is ${ }^{[2]}$ :

$$
G(z)=q^{2} \frac{\mu e^{i \theta}}{z-z_{c}}
$$

The dipole strength is $q, z_{c}$ is the position of the $c$-th obstacle, and $\mu e^{i \theta}$ is the velocity vector of the moving obstacle.

\subsection{Conformal Mapping Expands the Range of Obstacles}

Map the complex plane of the circular function to another complex plane through the analytical function, and change the plane reset potential.

Airfoil Common formulas for circular conformal transformation ${ }^{[7]}$ :

$$
z=\frac{1}{2}\left(\zeta+\frac{c^{2}}{\zeta}\right)
$$


An arc-shaped airfoil is mapped to a radius $a$, the center of the circle is in the $\zeta$-plane of $z_{c}$, and the angle of attack is $\theta$. Chord length is $c$ and wing thickness is $t$. The complex potential of the flow after the $\zeta$-plane conformal transformation is:

$$
F(\zeta)=\frac{1}{2} \mu e^{i \theta}\left[\left(\zeta-z_{c}\right)+\frac{a^{2} e^{2 i \theta}}{\zeta-z_{c}}+\frac{\Gamma}{2 \pi i} \ln \left(\zeta-z_{c}\right)\right]
$$

among them $\zeta=z+\sqrt{z^{2}-c^{2}}$ and $a=\sqrt{c^{2}+t^{2}}$, the equation can be transformed into:

$$
F(z)=\mu e^{i \theta}\left[\left(z+\sqrt{z^{2}-c^{2}}-z_{c}\right)+\frac{a^{2} e^{2 i \theta}}{z+\sqrt{z^{2}-c^{2}}-z_{c}}\right]+\frac{\Gamma}{2 \pi i} \ln \left(z+\sqrt{z^{2}-c^{2}}-z_{c}\right)
$$

The magnitude $\Gamma$ of the loop is: $\Gamma=2 \pi a \mu \sin \left(\operatorname{tg}^{-1} \frac{t}{c}+\theta\right)$.

\subsection{Correcting the Complex Velocity Function}

Because the flow field is composed of source and sink, the complex velocity near the source point will be infinite and the application value will disappear. A correction function is introduced to make the complex velocity of the target point converge, and to avoid chase based on the complex potential of the changed dynamic obstacle. The correction function is as follows ${ }^{[1]}$ :

$$
\begin{aligned}
& P_{H}(z)=\left|z-z_{k}\right|\left(1-e^{-k\left|z-z_{k}\right|}+\varepsilon\right) \\
& P_{F}(z)=\left\{\begin{array}{lr}
1 & \angle\left(z-z_{c}\right) \in\left[\theta-\frac{\pi}{2}, \theta+\frac{\pi}{2}\right] \\
\cos ^{2}(\varphi) & \angle\left(z-z_{c}\right) \in\left(\theta+\frac{\pi}{2}, \theta+\beta\right)
\end{array}\right.
\end{aligned}
$$

$\varphi$ is the difference between the angle of the moving obstacle with respect to the $x$-axis and the angle of the velocity vector. $\varepsilon$ is a small rational number, and $k$ is the deceleration coefficient after the particle is close to the target point. After introducing the correction function, the complete flow field complex velocity to avoid chasing can be written as ${ }^{[1]}$ :

$$
V_{j c}=P_{H}(z) H_{j}^{\prime}(z)+P_{F}(z)\left[F_{c}^{\prime}(z)+\frac{\left(d \mu e^{i \theta}\right)^{2}}{F_{c}^{\prime}(z)}\right]
$$

Among them, $H_{j}^{\prime}(z)$ and $F_{c}^{\prime}(z)$ are the derivatives of the $j$-th static obstacle $H_{j}(z)$ and the $c$-th dynamic obstacle $F_{c}(z)$, respectively.

\section{Obstacle Recovery Potential and Speed Stack ${ }^{[8]}$}

When the particles are at different positions, they are not affected by the impact of each obstacle. It is necessary to add a weight factor when multiple obstacles are superimposed to balance the force of the particles. The weight function $\delta_{j c}(z)$ determined according to the position of the particle is as follows:

$$
\boldsymbol{\delta}_{j c}(z)=\frac{\frac{1}{\left|z-z_{j c}\right|-R_{j c}}}{\sum_{k=1}^{n} \frac{1}{\left|z-z_{k}\right|-R_{j c}}}
$$

The weight ranges from 0 to 1 . The weight of the first obstacle is generally set to 1 . The compound speed after all obstacles are superimposed is:

$$
V_{j c}=\sum_{j c=1}^{n} \delta_{j c}(z)\left\{\left\{P_{H}(z) H_{j}^{\prime}(z)+P_{F}(z)\left[F_{c}^{\prime}(z)+\frac{\left(d \mu e^{i \theta}\right)^{2}}{F_{c}^{\prime}(z)}\right]\right\}\right\}
$$

After the function is constructed, it can more effectively avoid moving obstacles and escape the local minimum in advance. Determine the priority and weight, analyze the size of the particle affected by 
different obstacles, and plan the optimal trajectory of the particle.

\section{Location and Conditions Generated by the Virtual Target Source}

When facing U-shaped and C-shaped fixed obstacles, the particles tend to fall into the trap area, and the multiple speeds are zero, and multiple target points are required to guide the particles out of the area step by step. The application of additional target points requires prioritization of whether the particle is affected by the trap area. There are two main situations:

(1) When the particle is located inside, as shown in Figure 3, the particle is located in the area of the line connecting the end and end of the obstacle.

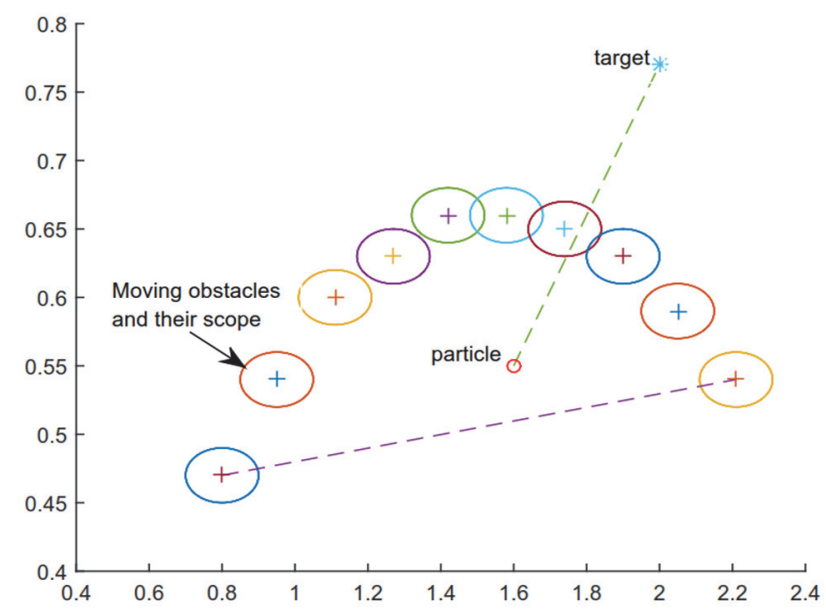

Figure 3. Inside the barrier

(2) When the particle is located outside, as shown in Figure 4, the particle is outside the area of the line connecting the end and end of the obstacle. The angle between the line connecting the obstacle and the target point is greater than the angle between the line connecting the target point and the particle.

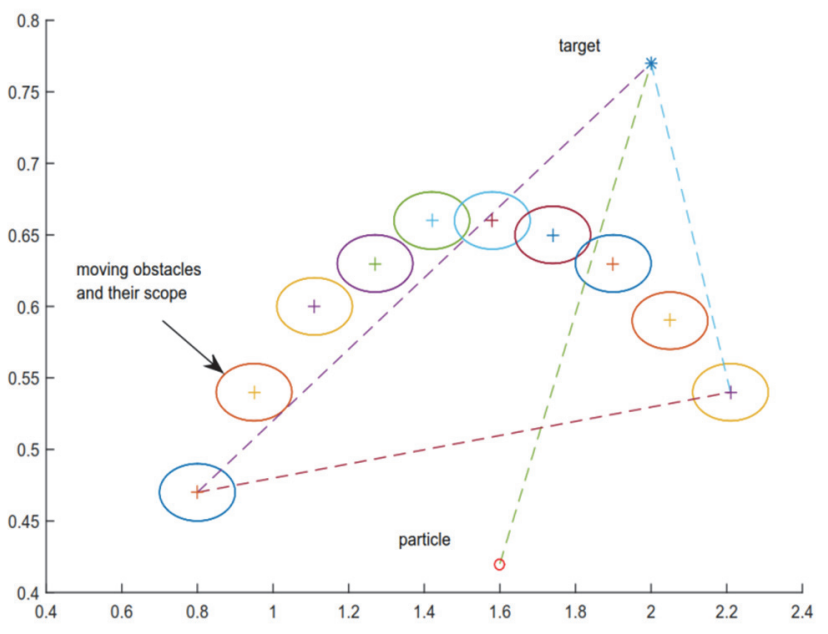

Figure 4. Outside the barrier

If the particles are in the above two cases, a virtual target point is set up to help the particles escape the area. The establishment method is as follows: 
When the number of connected obstacles is more than three, it is determined as a trap area. Record the coordinates $X, X_{0}$, and $X_{n}$ of the particle and the head or tail obstacle. Taking the coordinates of the head and tail obstacles as the center of symmetry, the symmetry point $X_{0 S}$ or $X_{n S}$ of the particle's current coordinate $X$ is made, and the resulting symmetry point is the virtual target point at the current time. The position of the virtual target point is shown in Figure 5:

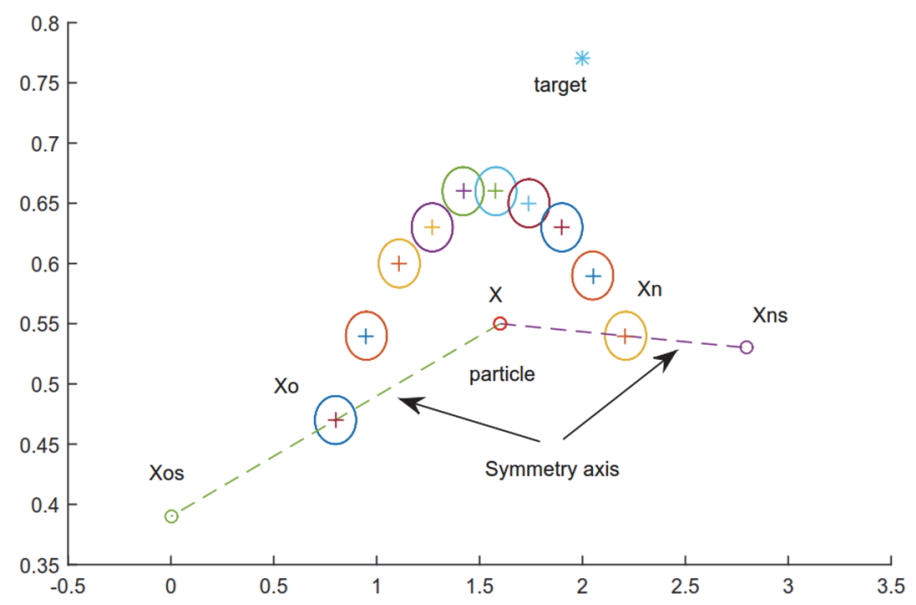

Figure 5. Virtual target point position distribution

The temporarily set up virtual target point only has a complex potential when it is trapped in the trap area, instead of the final target point step by step to guide the particle's course of action.

\section{Simulation and Analysis}

Set the environment variables in the flow field, including: fixed obstacles, moving airfoil obstacles, mass points, and target points. The particles move at the corresponding complex speed under the improved flow field repotential, smoothly avoiding obstacles and escaping from the trap area, planning: a safe and smooth path. As shown in Figure 6:

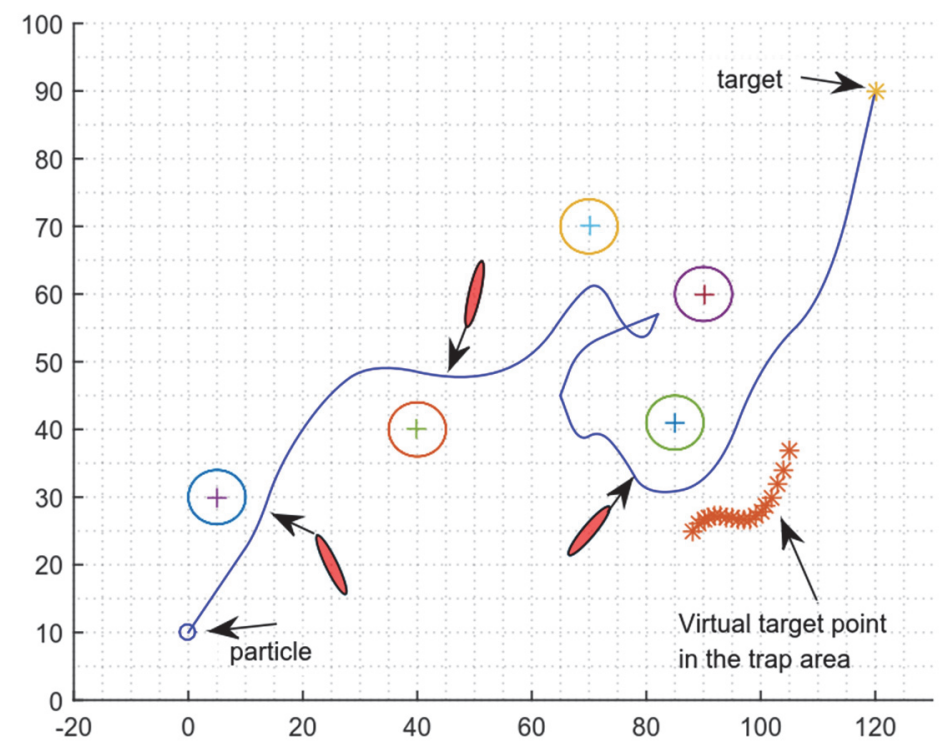

Figure 6. Obstacle avoidance of particles in complex potential field 


\section{Conclusion}

The traditional artificial potential field method uses the combined force to solve the obstacle avoidance problem of the object. There are mainly trap areas and local minimum problems. Therefore, the unique phenomenon in the flow field theory is used to change the complex potential function through eddy current and conformal transformation to replace the role of the repulsive force field, while improving the object's ability to perceive moving obstacles. Instead of the role of the gravitational field, the existence of the virtual target point avoids U and C-type traps, reduces unnecessary obstacle repulsion, and plans a smooth path faster. The introduction of the correction function eliminates the chasing domain of moving obstacles to a certain extent, and brings into the complex potential function the optimized velocity vector.

Acknowledgements. The work of this paper is supported by Guangxi Key Laboratory of Precision Navigation Technology and Application, Guilin University of Electronic Technology (No.DH201911). A special acknowledgement should give to Southwest Minzu University and Guilin University of Electronic Technology for its experimental conditions and technical support.

\section{References}

1. Shi Weiren, Huang Xinghua, Zhou Wei.Path planning of mobile robot based on improved artificial potential field method [J].Computer Applications, 2010,30 (8): 2021- 2023.

2. Yao, P., Wang, H. L. Three-dimensional path planning for UAV based on improved interfered fluid dynamical system and grey wolf optimizer[J]. Journal of the Optical Society of America B Optical Physics, 2016, 30(3):615. In Chinese.

3. Macktoobian, M., Shoorehdeli, M. A. Time-variant artificial potential field (TAPF): a breakthrough in power-optimized motion planning of autonomous space mobile robots[J]. Robotica, 2016, 34(5):1-23.

4. Qureshi, A. H., Ayaz, Y. Potential functions based sampling heuristic for optimal path planning $[\mathrm{J}]$. Autonomous Robots, 2015:1-15.

5. WANG Fujun. Computational fluid dynamics analysis [M]. Beijing: Tsinghua University Press, 2004: 81-83.

6. SHI R J, FAN X C, HE Y. Comprehensive evaluation index system for wind power utilization levels in wind farms in China [J]. Renewable \& sustainable energy reviews,2017,69: 461-471.

7. Dai,J. Y.,Wang, S.Y.,Yin,L.F.,et al. Hierarchical potential field algorithm of path planning for aircraft[J]. Control Theory \& Applications, 2015,32(11):1505-1510. In Chinese.

8. DU TOIT N E, BURDICK J W. Robotic motion planning in dynamic, cluttered, uncertain environments [C]// 2010 IEEE International Conference on Robotics and Automation. Anchorage: IEEE, 2010: 966-973.

9. Zhang, D. F., Liu, F. Research and development trend of path planning based on artificial potential field method[J]. Computer Engineering \& Science, 2013, 35(6):88-95. In Chinese.

10. Kovács, B., Szayer, G., et al. A novel potential field method for path planning of mobile robots by adapting animal motion attributes[J]. Robotics \& Autonomous Systems, 2016, 82(C):24-34..

11. CHENG Zhi, ZHANG Zhi'an, LI Jinzhi, et al. Mobile robots path planning based on improved artificial potential field[J]. Computer Engineering and Applications,2019.

12. Xiaoping HU, Zeyu LI, Jing CAO. A Path Planning Method Based on Artificial Potential Field Improved by Potential Flow Theory[J]. International Conference on Computer Science and Technology,2017. 